\title{
The Issue of Bleeding from The Carotid Arteries
}

\author{
Shaposhnikov Veniamin Ivanovich* \\ Professor of surgical diseases, Russia

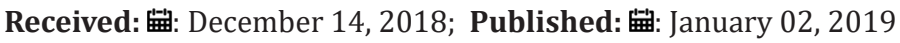 \\ *Corresponding author: Shaposhnikov Veniamin Ivanovich, Professor of surgical diseases, Russia
}

\begin{abstract}
The author describes the two informative clinical observations of bleeding from the carotid arteries. One of them was iatrogenic in nature and occurred during surgery for chronic tonsillitis. The patient was an anomaly of the internal carotid artery passing through the amygdala and to manipulate the surgeon, seriously injured her. Had to produce this ligation arteries that could lead to disruption of blood circulation in the brain. Fortunately, this did not happen. The second observation for health reasons was bandaged external carotid artery. The cause of the bleeding was systemic lupus red nose. Both observations are among the quibbling.
\end{abstract}

Keywords: Bleeding; Carotid arteries; Trauma; Tissue Necrosis of Nose

\section{Introduction}

Bleeding from hundredths of arteries is among the serious complications that require emergency surgery. If they are damaged superimposed vascular suture. However, a defect sometimes impossible to weld overlay the walls and has them dress for. If this manipulation is done on the external carotid artery, cerebrovascular accidents do not usually occur. If optionally internal carotid artery, it can lead to anemia in the brain. Anticipate this complication is impossible and the surgeon is forced to take risks, to save the life of ancients.

\section{Surveillance 1}

The girl came into the Office on a scheduled tonsillectomy. The operation was recommended rheumatologist. On examination, the throats except for increase in size of the tonsils other pathologies are detected. The operation became an experienced professional who has seniority Otolaryngologist for over twenty years. They decided to perform a subtotal tonsillectomy. Perednebokovyh. Office parts left tonsil from the mucous membranes of the pharynx are observed normal bleeding. Easily managed to get the loop tonsillectomy around the removed tissues, and when the manipulation was performed, from wounds Zev hit jet of scarlet blood. The patient became to choke her. There was panic in the operating room. At this time, I accidentally was in neighboring operating. Noise and screams have alerted me, and I decided to find out the cause of the panic. Nature of bleeding testified that this girl was an anomalous internal carotid artery passing through the tissue behind the tonsils (in close contact with them) and subtotal resection of the damage occurred this large vessel.

Immediately was made a typical finger pressure common carotid artery, and tracheal intubation tube with an inflatable cuff. General anesthesia is started. Of the trachea and bronchi was removed the liquid blood and it clots. The threat of continued development of aspiration pneumonia (Mendelssohn syndrome). After tracheal intubations Otolaryngologist performed tight stopped the oral cavity and pharynx, it was found that the rugged vessel was reduced and shifted into the tissues of the neck. The situation was simply criminal-girl needed a wide incision on the neck, and this caused cosmetic discomfort and another way to meet the continuing stop bleeding was impossible. Moreover, these ligation arteries could lead to a violation of cerebral circulation, which could end with her death or mental disability. Surgery was required, and its outcome was just unpredictable.

Doctors took place. It was decided to operate. The patient's mother was set up very aggressively but gave consent to the operation. And in such situations had to do surgery-internal carotid artery ligation on her over (via Askew-longitudinal access at the neck in the course of the stenciled-mastoid muscles). The operation and the postoperative period were uneventful. The bleeding has been stopped, while the mental activity the patient did not suffer. Such a favorable outcome of the operation can be explained only by the fact that the very nature of vascular pathology in this patient 
was accompanied by the development of her collateral cerebral circulation. This was due to the fact that the frequent exacerbations of chronic tonsillitis caused compression of this vessel inflammatory infiltration that contributed to the development of collaterals. Oneyear check-up showed that she practically healthy. It was explained the reason for her scar on the body. Surgeons claims she didn't have.

\section{Surveillance 2}

He entered office a man 37 years with arterial bleeding from the nose. During the 10 years suffered allergic Vasculitis (Systemic Lupus Erythematosus). Specialists have been treated consistently in various hospitals of the country. The last time such treatment took place 2 months ago. Treatment was horizontal, mainly with corticosteroids. When the primary examination of the patient on the pale of swelling and outlasted his face was visible Nodular necrotic tumor, which is more thrilling to the left half of the nose. From the left nasal passage stood the tampon is the emergency doctor tried to do the front stopped nose. The patient constantly had done swallowing motions. It was clear that he was choking with blood. Was done urgently back and then stiff front tamponade. It was clear that the cravat branches of the external carotid artery and left Nasal packing may not reliably stop bleeding. To do this required lefthanging barrel ligation of the external carotid artery in the neck. On the right was said to the patient. He consented to the operation. It was clear that the cravat branches of the external carotid artery and left Nasal packing may not reliably stop bleeding. To do this required left-hanging barrel ligation of the external carotid artery in the neck. On the right was said to the patient. He consented to the operation. Because of hormonal status, it was decided to run under local anesthesia. Oblique incision length of $14 \mathrm{~cm}$ is made on the left front edge of the sternocleidomastoid muscle. Without her was common carotid artery, and then outside. It was determined by the side of orthodontic vessels. Artery twice was bandaged, and stunt doubled stitched and bandaged [1-4].

The intersection of the vessel carried out between a pair of ligatures. This caution was caused by decrease of reoperation processes, which connected both with the disease itself, and hormone therapy. Post-operative period was uneventful. Nosebleed stopped immediately after the operation and stopped nose stopped. The patient was dis-charged from Office in two weeks. Visually the swelling in the face by this time decreased in size and not bleeding. Through 3 months check-up showed that the tumor continues to shrink in size. This positive process clearly was due to reduced blood flow to injured tissues.

\section{Discussion}

The two observations, in fact, can occur in any medical institution, and for this reason are relevant on the issue of information colleagues surf right decision if something similar happens. Anecdotal observations only underscore the complexity of the profession of surgeon in saving the patient's life. It is necessary to have a wide range of professional skills to make the right standard solution and succeed in a seemingly hopeless situation. To compile these observations is difficult or even impossible, since each observation is unique and deserves attention.

\section{Conclusion}

Bleeding from the carotid arteries is among the most severe complications. You want fast reaction on finding reliable ways to remedy it. Temporary stopping hemorrhages techniques allow only to prepare for the substantive and technical event-ligation of the internal or external carotid artery. This manipulation in these cases does not result in a breach of the blood circulation in the brain, which can be explained by the slow growing vessel compression in development of the disease. In one observation it was chronic tonsillitis and another Systemic Lupus Erythematosus.

\section{References}

1. Rabotnikov VS, Spiridonov AA Vessels (1988) Clinical surgery Reference guide for doctors under the editorship of YM Pancyreva. Moscow medicine, pp. 444-447.

2. Sokolov VA (2006) Multiple and associated injuries/VA Sokolov M: GJeOT AR medicine.

3. Yumashev GS (1987) Traumatology and orthopedics M: 357.

4. Shaposhnikov Vi (2008) Injury prevention. Krasnodar p. 48.
ISSN: 2574-1241

DOI: $10.26717 /$ BJSTR.2019.12.002288

Shaposhnikov Veniamin Ivanovich. Biomed J Sci \& Tech Res

This work is licensed under Creative Commons Attribution 4.0 License

Submission Link: https://biomedres.us/submit-manuscript.php

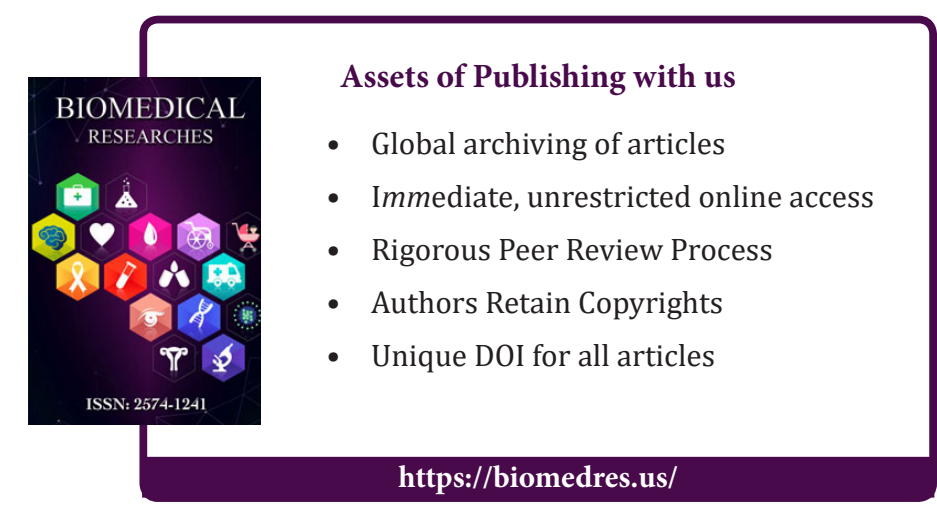

